\title{
COL6A6 inhibits the proliferation and metastasis of non-small cell lung cancer through the JAK signalling pathway
}

\author{
Han Qiao, Yan Feng, Huaping Tang \\ Department of Respiratory Medicine, Qingdao Municipal Hospital, Qingdao University, Qingdao, China \\ Contributions: (I) Conception and design: All authors; (II) Administrative support: H Qiao, H Tang; (III) Provision of study materials or patients: \\ H Qiao, H Tang; (IV) Collection and assembly of data: All authors; (V) Data analysis and interpretation: All authors; (VI) Manuscript writing: All \\ authors; (VII) Final approval of manuscript: All authors. \\ Correspondence to: Huaping Tang. Department of Respiratory Medicine, Qingdao Municipal Hospital, Qingdao 266071, China. Email: qdthp@126.com.
}

\begin{abstract}
Background: Collagen type VI alpha 6 chain (COL6A6) plays a vital role in maintaining cell structural integrity and regulating cell function. It has been proven to be a tumor suppressor gene and molecular therapeutic target. However, the mechanism of COL6A6 in non-small cell lung cancer (NSCLC) has not been elucidated. The purpose of this study was to investigate the relationship between COL6A6 and NSCLC.

Methods: We analyzed the expression of COL6A6 in NSCLC using public databases and verified the findings in NSCLC tissues and cells. The protein expression of COL6A6 was evaluated by Western blot. The CCK8 and Transwell assays were used to assess the invasion ability of NSCLC cells after COL6A6 knockdown. At the same time, we discussed the role of the JAK signalling pathway that may be related to COL6A6.
\end{abstract}

Results: Bioinformatics analysis showed that COL6A6 expression was downregulated in NSCLC, and its high expression was associated with a better prognosis of NSCLC. In vitro, the expression of COL6A6 in NSCLC tissues was significantly lower than that in adjacent tissues. Furthermore, COL6A6 knockout accelerated the proliferation, invasion, and migration of NSCLC cells and activated the JAK signalling pathway.

Conclusions: Our study illustrates that COL6A6 is a tumor suppressor gene in NSCLC and is involved in NSCLC tumorigenesis by regulating the JAK signalling pathway. Therefore, COL6A6 holds promise as a molecular therapeutic target for NSCLC.

Keywords: Collagen type VI alpha 6 chain (COL6A6); non-small cell lung cancer (NSCLC); proliferation; invasion; JAK signalling

Submitted Aug 22, 2021. Accepted for publication Oct 16, 2021.

doi: $10.21037 /$ tcr-21-2002

View this article at: https://dx.doi.org/10.21037/tcr-21-2002

\section{Introduction}

Lung cancer is the most common cancer globally and the leading cause of cancer death $(1,2)$. Non-small cell lung cancer (NSCLC) accounts for most lung cancer types, including squamous cell carcinoma, adenocarcinoma, and large cell carcinoma (3). Although tumor molecular biology, genomics, targeted therapy, and other fields have been developing in recent years, the average 5 -year survival rate of most patients with advanced NSCLC is only 19\% (4-6). Notably, tumorigenesis may be significantly related to the change of the essential genes $(7,8)$. Therefore, it is imperative to explore the pathogenesis of NSCLC and seek new therapeutic targets for early diagnosis and to improve patients' survival rates.

Collagen type VI alpha 6 chain (COL6A6) belongs to the family of collagen type VI (COL6), which is encoded by a 6,789-bp coding region. As an extracellular matrix protein, 
COL6A6 is significantly expressed in various human tissues, including lung, kidney, liver, spleen, thymus, heart, and skeletal muscle, and plays an essential role in maintaining cell structure integrity and regulating cell function $(9,10)$. Long et al. found that COL6A6 can suppress the growth and metastasis of pituitary adenoma (11). Yeh et al. confirmed that high expression of COL6A6 has a significant relationship with the early pathological stage of breast cancer (12). In addition, many COL6 family members have been shown to play essential roles in tumor initiation and progression (13-15). However, it is not clear whether COL6A6 is associated with NSCLC.

Our previous analysis using public databases [Gene Expression Profiling Interactive Analysis (GEPIA) and The Cancer Genome Atlas (TCGA)] showed that COL6A6 mRNA was significantly reduced in lung adenocarcinoma (LUAD) and lung squamous cell carcinoma (LUSC). Therefore, we hypothesized that COL6A6 plays a critical role in NSCLC. TCGA database was used to analyze the correlation between COL6A6 and the NSCLC survival. After that, the COL6A6 protein level was verified in NSCLC tissues and cell lines. It was confirmed that COL6A6 participates in the proliferation and metastasis of NSCLC cells through cell transfection methods. Finally, gene set enrichment analysis (GSEA) datasets and Western blotting demonstrated that COL6A6 is mainly associated with the JAK signalling pathway. Therefore, the COL6A6 gene can serve as a potentially valuable prognostic biomarker and therapeutic target for NSCLC. We present the following article in accordance with the MDAR reporting checklist (available at https://dx.doi.org/10.21037/tcr-21-2002).

\section{Methods}

\section{Bioinformatics prediction}

We obtained data from TCGA database (https:// cancergenome.nih.gov/) and the GEPIA website (http:// gepia.cancer-pku.cn/) on the COL6A6 mRNA sequences in NSCLC tissue specimens and adjacent lung tissues. At the same time, we carried out GSEA using the gsea-4.0.jar website (https://www.gsea-msigdb.org/gsea/index.jsp). Kaplan-Meier analysis estimated the survival of NSCLC patients who showed diverse expression levels of COL6A6 (http://kmplot.com/analysis/).

\section{Patients and tissue specimens}

From January to December 2020, with the approval of the medical ethics committee of Qingdao Municipal Hospital Affiliated to Qingdao University (2021yxy32) and the informed consent of patients, we collected 20 pairs of tumor tissues and adjacent healthy tissues of lung cancer patients in Qingdao Municipal Hospital. The study was conducted in accordance with the Declaration of Helsinki (as revised in 2013).

\section{Cell lines and culture}

The immortalized bronchial epithelial cell line (BEAS-2B) and 4 NSCLC cell lines (H520, A549, H1299, and H1975) were all purchased from Procell Life Science \& Technology Co., Ltd. (Wuhan, China). Cells were maintained in medium containing 10\% fetal bovine serum (FBS) (10091148, GIBCO; Shanghai, China) and $1 \%$ penicillinstreptomycin solution, and placed in a cell incubator at $37^{\circ} \mathrm{C}$ and $5 \% \mathrm{CO}_{2}$.

\section{Cell transfection and grouping}

COL6A6-siRNA was purchased from Shanghai GenePharma Co., Ltd. H1299 cells in good condition were transferred to a 6-well plate in advance. Then, $10 \mu \mathrm{L}$ COL6A6-siRNA was diluted with $100 \mu \mathrm{L}$ OPTI-MEM (\#31985, GIBCO) and added to $5 \mu \mathrm{L}$ Lipofectamine ${ }^{\mathrm{TM}}$ 2000 (\#11668-019, Invitrogen; Shanghai, China) in $100 \mu \mathrm{L}$ OPTI-MEM, and was left to stand at room temperature for 20 minutes. A total of $200 \mu \mathrm{L}$ of mixture was added to each well. After 6 hours in the cell incubator, the mixture was aspirated out and replaced with standard medium. At 48 hours later, the cells were then harvested for subsequent experiments.

\section{$R T-q P C R$}

After the cellular RNA was extracted with Trizol reagent (15596-026, Ambion; Shanghai, China), the light absorption value was measured using a microspectrophotometer (Nano-100, China) to calculate the purity and concentration of RNA. The RNA quality was estimated according to the OD260/OD280 ratio, which was required to be between $1.8-2.0$ to meet the experimental requirements. The isolated total RNA was reverse transcribed into cDNA and amplified using the SYBR-Green Master Mix (Q111-02, Vzyme; Nanjing, China). The primers used in this study included: COL6A6: forward 5'-AAGTGGTGCTTAGGGGTTC-3'; reverse 
5'-CCAACAATTAACAAAAGAAAGG-3'. GAPDH was used as an internal control, and its primers were as follows: forward 5'-TCAAGAAGGTGGTGAAGCAGG-3' and reverse 5'-TCAAAGGTGGAGGAGTGGGT-3'. The $2^{-\Delta \Delta C t}$ method was used to calculate the relative mRNA expression levels.

\section{Western blot analysis}

Western blotting was performed according to standard methods as described previously (16), using anti-COL6A6 (1:1,000, PA5-60958, Invitrogen), anti-JAK2 (1:1,000, AF6023, Affinity; Changzhou, China), anti-p-JAK2 (1:1,000, AF3024, Affinity), anti-STAT3 (1:1,000, 10253-2-AP; Proteintech; Wuhan, China), and anti-p-STAT3 (1:2,000, Ab76315, Abcam; Cambridge, UK) antibodies. AntiGAPDH (1:2,000, AB-P-R001; Goodhere Biotechnology Co., Ltd.; Hangzhou, China) was used as the loading control.

\section{CCK8 assay}

The transfected H1299 cells were inoculated into 96well plates at $5 \times 10^{3}$ per well and cultured overnight in an incubator at $37^{\circ} \mathrm{C}$ with $5 \% \mathrm{CO}_{2}$. Then, $10 \mu \mathrm{L}$ CCK8 (HYK0301, MedChemExpress; Shanghai, China) was added to each well, and the OD value of $450 \mathrm{~nm}$ in each well was detected with an enzyme marker (Multiskan Skyhigh; Waltham, USA).

\section{Wound healing assay}

The transfected H1299 cells were seeded in a 6-well plate and incubated overnight in a $5 \% \mathrm{CO}_{2}$ incubator at $37{ }^{\circ} \mathrm{C}$. When the cells reached the bottom of the 6-well plate evenly, we used the tip of a sterilized pipette to scrape off the cell layer gently. The cells were washed with PBS 3 times, then serum-free medium was added. Cells were imaged under a microscope (IX51, Olympus, Japan) after 0 and 24 hours.

\section{Transwell invasion test}

The transfected H1299 cells were seeded in a 6-well plate at $5 \times 10^{5}$ per well, with $2 \mathrm{~mL}$ of cell suspension added to each well. Cells were incubated overnight in an incubator at $37^{\circ} \mathrm{C}$ and $5 \% \mathrm{CO}_{2}$. When the cell density reached $80-90 \%$, the cells were digested and sub-cultured with $0.25 \%$ trypsin and resuspended with serum-free medium. The Matrigel (356234, Corning; New York, USA) melted at $4{ }^{\circ} \mathrm{C}$ and was diluted in serum-free medium to a final concentration of $1 \mathrm{mg} / \mathrm{mL}$. Then, we added $800 \mu \mathrm{L}$ medium (cell culture medium plus $10 \%$ FBS) to the 24 -well plate and put it into the Transwell chamber, then put $100 \mu \mathrm{L}$ Matrigel in the Transwell upper chamber. After drying and forming a gel, cell suspensions of each group were inoculated in the Transwell for 24 hours. The cells were fixed with $70 \%$ ice cold ethanol solution for 1 hour, then stained with $0.5 \%$ crystal violet dye and photographed under a microscope (IX51, Olympus, Japan).

\section{Statistical analysis}

The Data was performed using GraphPad Prism 5 software, and a $\mathrm{P}$ value less than 0.05 was considered statistically significant. The overall survival rates were calculated using Kaplan-Meier method. Paired $t$-test or one-way ANOVA analysis of variance were used for comparisons between groups. Data are presented as mean \pm standard deviation. All independent experiments were performed at least 3 times.

\section{Results}

\section{The expression of COL6A6 is downregulated in NSCLC}

Based on the tumor databases GEPIA and TCGA, we found that COL6A6 expression was significantly reduced in NSCLC tissues compared with healthy lung tissues (Figure $1 \mathrm{~A}, 1 \mathrm{~B})$. At the same time, we verified this conclusion with Western blotting (Figure 1C). We further compared the expression levels between the BEAS-2B cell line and the 4 NSCLC cell lines (A549, H1299, H1975, H520). COL6A6 expression in NSCLC cells was significantly reduced compared to that in the BEAS-2B cell line (Figure 1D). All the results demonstrate that COL6A6 expression is downregulated in NSCLC cells and tissues. In addition, the Kaplan-Meier plotter was used to analyze the correlation between COL6A6 expression and the NSCLC survival. The results showed that the lower the COL6A6 expression, the worse the prognosis of patients with lung cancer (Figure 1E).

\section{COL6A6 inbibits NSCLC cells proliferation and invasion}

COL6A6 expression in the H1299 cell line was higher 
A

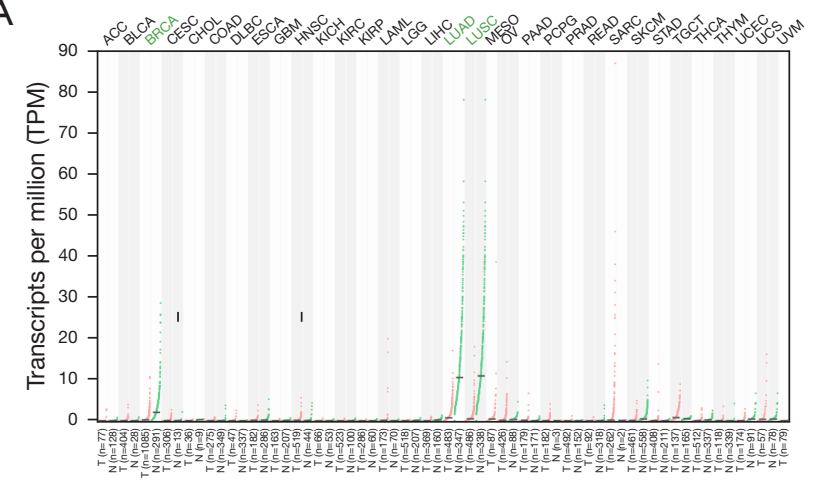

B

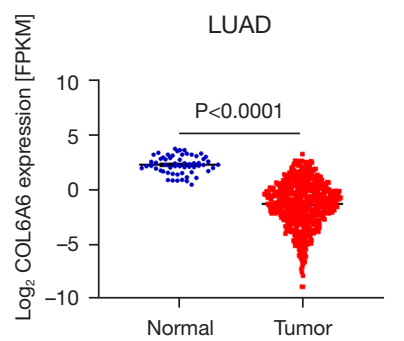

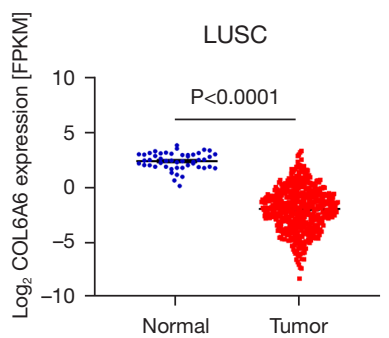

C

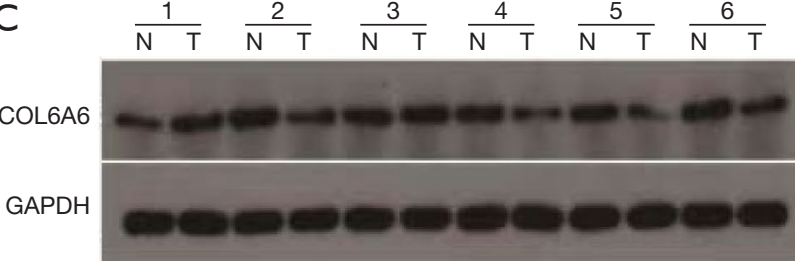

Ratio

$\begin{array}{llllllllllll}1 & 2.14 & 1 & 0.43 & 1 & 1.18 & 1 & 0.39 & 1 & 0.45 & 1 & 0.57\end{array}$

$\frac{7}{\mathrm{~N} T} \frac{8}{\mathrm{~N} T} \frac{9}{\mathrm{~N} T} \quad \frac{10}{\mathrm{~N} T} \quad \frac{11}{\mathrm{~N} T} \frac{12}{\mathrm{~N} T}$

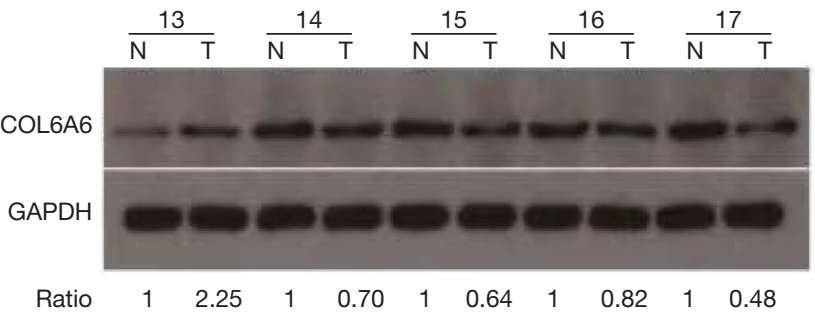

D
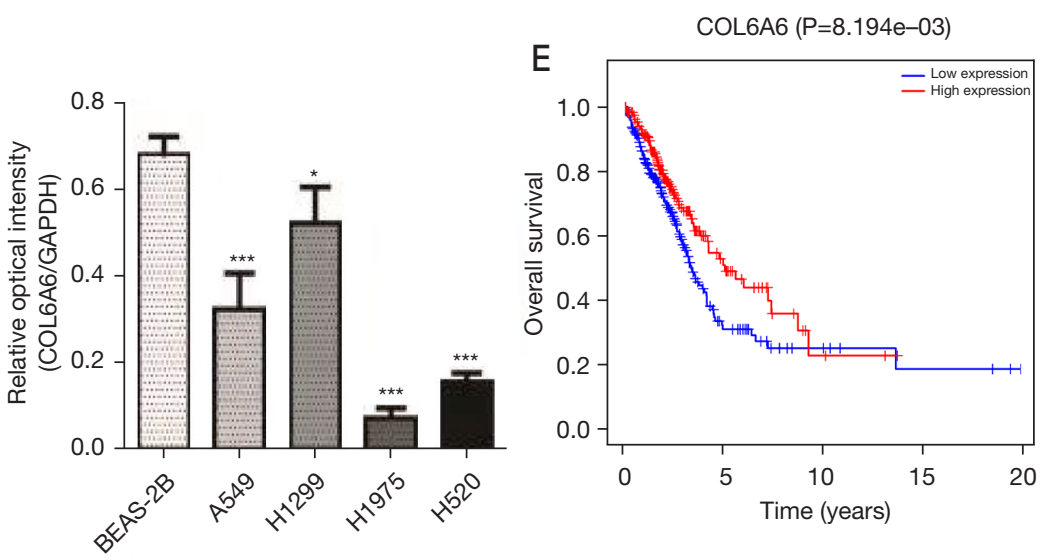

Figure 1 The expression of COL6A6 in NSCLC tissues. (A) COL6A6 is differentially expressed in tumors compared to normal tissues according to the GEPIA database. (B) The expression of COL6A6 in LUAD and LUSC in TCGA database $(\mathrm{P}<0.001)$. (C) Western blotting analysis of COL6A6 in NSCLC and adjacent tissues was performed. (D) The expression of COL6A6 in the immortalized bronchial epithelial cell line (BEAS-2B) and 4 NSCLC cell lines (H520, A549, H1299, and H1975) was analyzed by Western blotting. (E) The Kaplan-Meier plotter was used to analyze the overall survival, which showed that the prognosis of patients with high COL6A6 expression (red) was better than that of patients with low COL6A6 expression (blue). Data are expressed as mean $\pm \mathrm{SE}$. *, $\mathrm{P}<0.05$; ${ }^{* *}, \mathrm{P}<0.001$. N, normal tissue; T, tumor tissue; COL6A6, collagen type VI alpha 6 chain; GAPDH, glyceraldehyde 3-phosphate dehydrogenase; TCGA, The Cancer Genome Atlas; GEPIA, Gene Expression Profiling Interactive Analysis; LUAD, lung adenocarcinoma; LUSC, lung squamous cell carcinoma; NSCLC, non-small cell lung cancer; SE, standard error. 
A

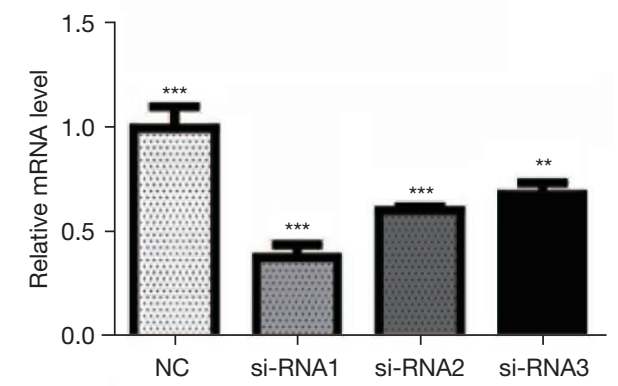

C

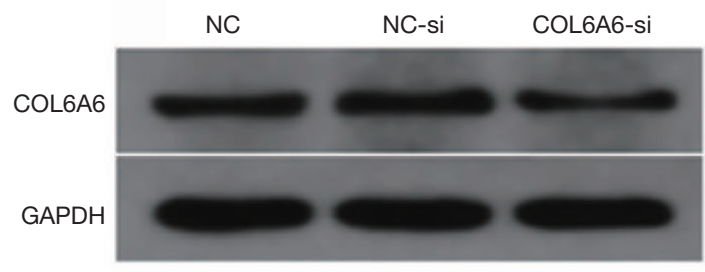

B
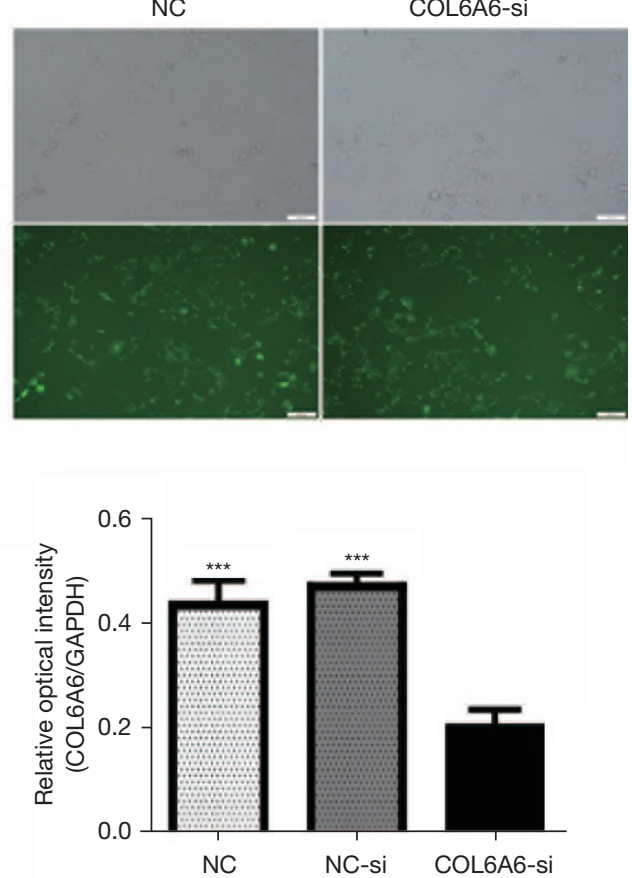

Figure 2 Verification of the transfection efficiency of COL6A6 knockdown in NSCLC cells. (A) Lentiviral siRNA targeting COL6A6 was transfected into $\mathrm{H} 1299$ cells. (B) More than $80 \%$ of the cells were green fluorescent protein (GFP) positive and were transfected successfully (×100). Scale bar: $100 \mu \mathrm{m}$. (C) Western blotting assays were used to detect the expression of COL6A6 in H1299 cells transfected with siRNA. NC: uninfected H1299 cells; NC-si: negative control siRNA transfected H1299 cells; COL6A6-si: COL6A6 siRNA transfected H1299 cells. Data are expressed as mean $\pm \mathrm{SE}$. ${ }^{* *}, \mathrm{P}<0.01$; ${ }^{* *}, \mathrm{P}<0.001$. COL6A6, collagen type VI alpha 6 chain; NSCLC, non-small cell lung cancer; SE, standard error.

than that in the other NSCLC cell lines (Figure 1D). We transfected COL6A6-siRNA into H1299 cells and analyzed the expression level of COL6A6 by Western blotting. The results showed that COL6A6-siRNA significantly inhibited the expression level of COL6A6 in the H1299 cell line (Figure 2A-2C). To further investigate the role of COL6A6 in NSCLC, the CCK8 assay was performed to evaluate cell proliferation. The CCK8 assay showed that the inhibition of COL6A6 significantly promoted the proliferation of H1299 cells (Figure 3A). Then, the effect of COL6A6 on the migration and invasion of NSCLC cells was verified by the wound healing assay and the Transwell assay. It was found that, in the COL6A6-siRNA group, the migration and metastasis ability of $\mathrm{H} 1299$ cells were notably enhanced than the control group (Figure 3B,3C).

\section{COL6A6 inhibits NSCLC progression through the $\mathcal{F A K}$ signalling pathway}

Finally, GSEA software was used to search for COL6A6- related signalling pathways to understand the specific regulatory role of COL6A6 in NSCLC. Our analysis suggests that COL6A6 may inhibit the progression of NSCLC through the JAK signalling pathway (Figure 4A). The phosphorylation levels of JAK and STAT in the COL6A6 knockout group were significantly higher than those in the control group (Figure 4B,4C). All the results clearly demonstrated that COL6A6 knockout could activate the JAK signalling pathway.

\section{Discussion}

Lung cancer is still the leading cause of cancer deaths globally due to a lack of understanding of the molecular mechanisms $(1,5)$. TNM stage has always been considered as the best determinant to judge the prognosis of NSCLC patients. With recent studies, people believe that cancer is not only an autonomous cell disease, but also forms a complex ecosystem, and its immune microenvironment can affect the growth, diffusion and prognosis of tumors. The 
A

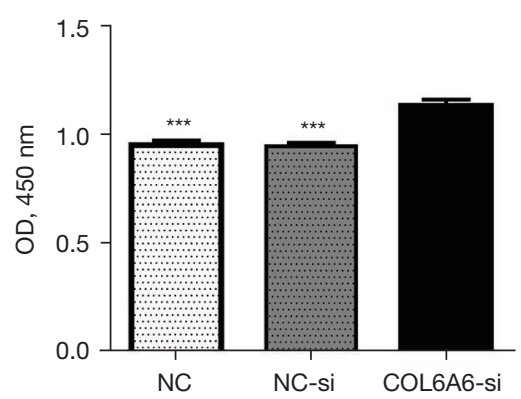

B

NC

NC-si

COL6A6-si
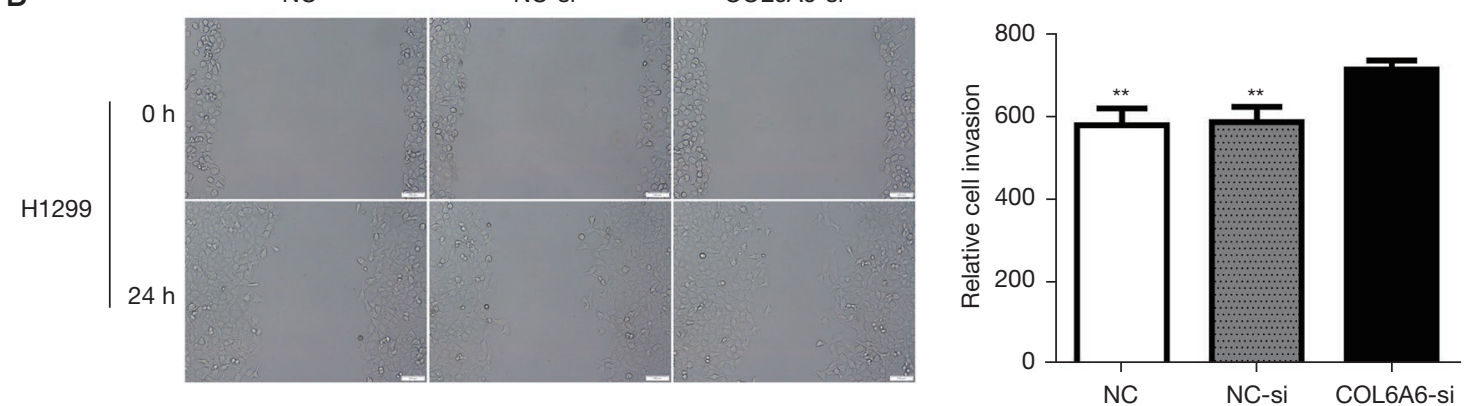

C
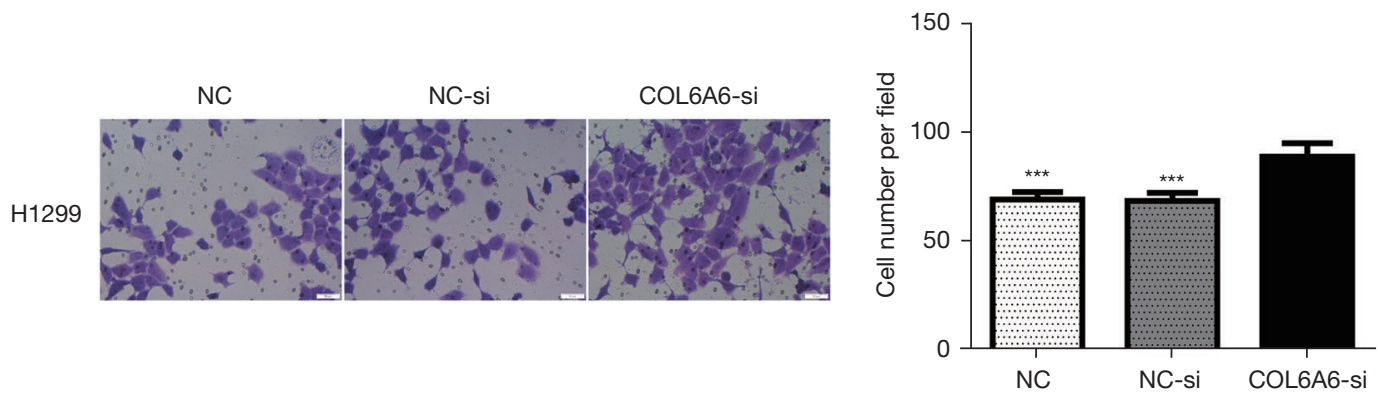

Figure 3 COL6A6 inhibits the proliferation and invasion of NSCLC cells. (A) The proliferation efficiency of NC, NC-si, and COL6A6si in H1299 cells after transfection was detected by CCK8 analysis. (B) the wound healing assay analyzed the migration of H1299 cells transfected with COL6A6-si (×100). Scale bar, $100 \mu \mathrm{m}$. (C) the number of invasive H1299 cells was detected by the Transwell assay (200x). The cells were stained with $0.5 \%$ crystal violet dye. Scale bar, $50 \mu \mathrm{m}$. NC: uninfected H1299 cells; NC-si: negative control siRNA transfected H1299 cells; COL6A6-si: COL6A6 siRNA transfected H1299 cells. ${ }^{* *}, \mathrm{P}<0.01$; ${ }^{* * *}, \mathrm{P}<0.001$. COL6A6, collagen type VI alpha 6 chain; NSCLC, non-small cell lung cancer.

interaction of tumor/host microenvironment promotes the development of prognostic and predictive biomarkers. In recent years, many studies have shown that some essential genes have a decisive influence on the occurrence and development of NSCLC. Therefore, in this study, we explored the relationship between COL6A6 and NSCLC. We found that COL6A6 expression was decreased in
NSCLC, and was associated with favorable prognostic factors in patients. In addition, low COL6A6 expression activated the JAK signalling pathway, suggesting that low COL6A6 expression effectively promotes NSCLC proliferation and metastasis. In conclusion, we identified for the first time the role of COL6A6 as a tumor-critical gene in NSCLC. 
A
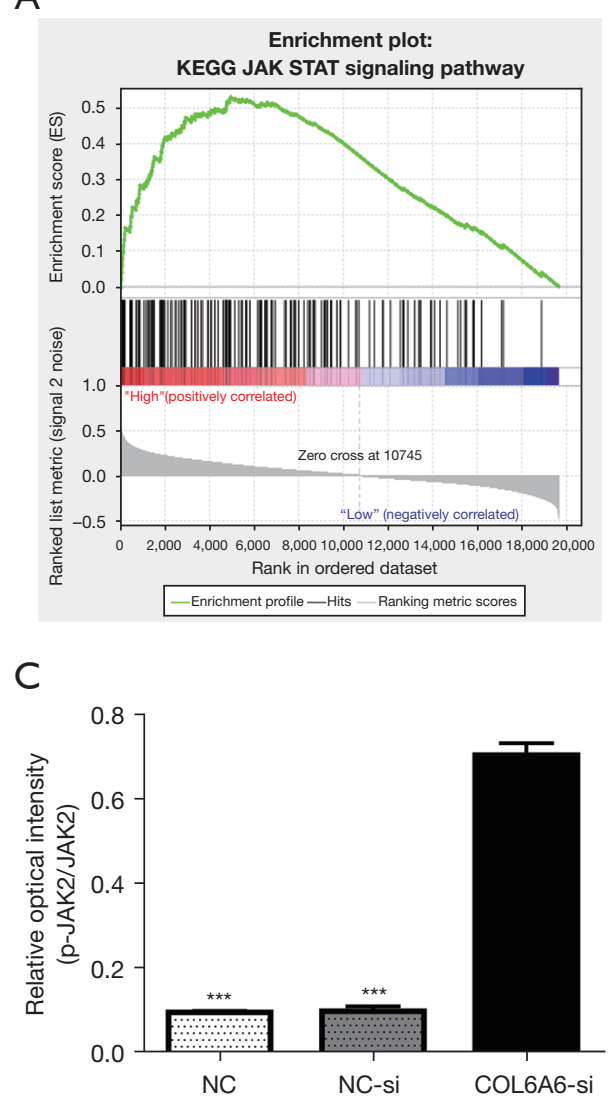

B
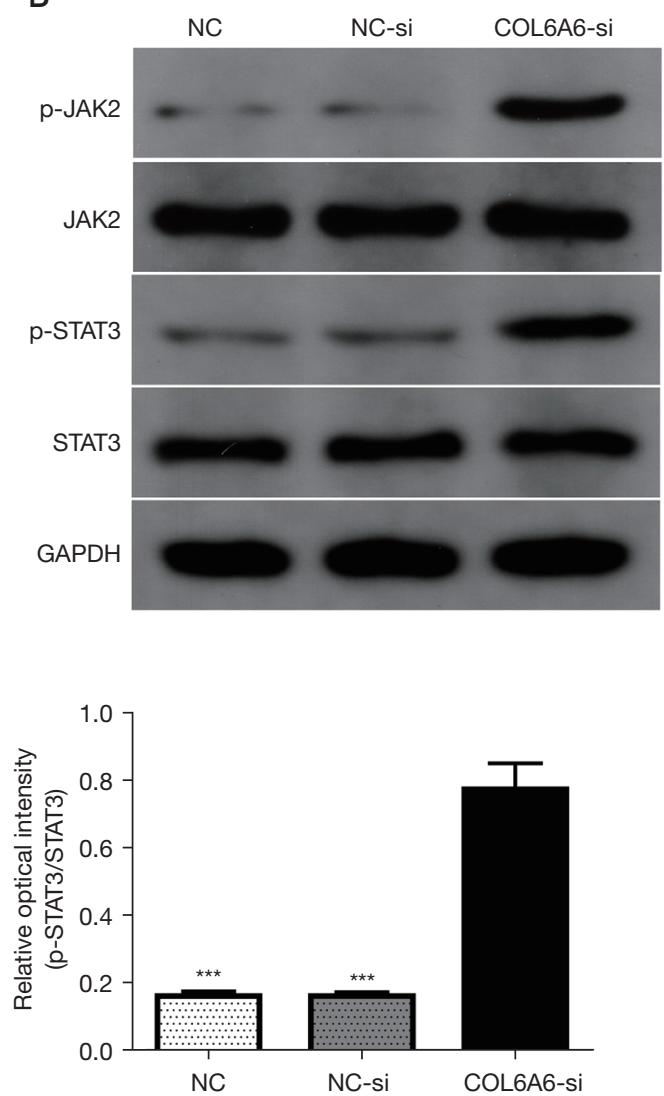

Figure 4 COL6A6 regulates the JAK signalling pathway to inhibit the progression of NSCLC. (A) GSEA demonstrated that COL6A6 is related to the JAK signalling pathway. (B,C) Western blotting assays for JAK-2, p-JAK2, STAT3, and p-STAT3 in H1299 cells following knockdown of COL6A6. NC: uninfected H1299 cells; NC-si: negative control siRNA transfected H1299 cells; COL6A6-si: COL6A6 siRNA transfected H1299 cells. ${ }^{* * *}, \mathrm{P}<0.001$. COL6A6, collagen type VI alpha 6 chain; GSEA, gene set enrichment analysis; NSCLC, nonsmall cell lung cancer.

The COL6 family plays a critical role in cell adhesion $(17,18)$, apoptosis, autophagy (19), and dystrophy (20). Interestingly, some members are involved in the growth and development of tumors $(21,22)$. In previous studies, COL6A6 has been shown to be a cancer suppressor gene that acts as an independent prognostic marker for pituitary adenomas. Long et al. reported that COL6A6 interacted with $\mathrm{P} 4 \mathrm{HA} 3$ to suppress the growth and metastasis of pituitary adenoma via blocking the PI3K-Akt pathway (11). However, the specific role of COL6A6 in NSCLC metastasis has not been confirmed until now, thus we conducted the following experimental analysis. First, we analyzed COL6A6 mRNA sequence data in NSCLC tissue samples to evaluate the survival of patients with different levels of COL6A6 expression using Kaplan-Meier analysis.
The results showed that COL6A6 expression was low in NSCLC, and its high expression was associated with a better prognosis of NSCLC. We then validated this conclusion with Western blot analysis, which showed that COL6A6 expression was significantly lower in NSCLC tissue samples than in normal lung tissues. The in vitro experiments (CCK8 assay, wound healing assay, and Transwell assay) showed that the proliferation and migration ability of NSCLC cells after COL6A6 knockdown was significantly enhanced. At the same time, the bioinformatics analysis showed that the higher the COL6A6 expression, the better the prognosis of patients. In conclusion, we hypothesized that COL6A6 is involved in the development and progression of NSCLC. The occurrence and progression of lung cancer is a multistep process. This process involves abnormal gene changes 
and protein expression, followed by cell phenotypic transformation and complex intracellular signalling pathways. In addition to the genetic characteristics of lung cancer, the need for protein biomarkers needs to be addressed to accurately subclassify lung cancer and monitor disease progression and treatment effect. Signalling through the JAK pathway is essential in the progression of colorectal cancer, gastric cancer, bladder carcinoma, and other tumors $(23-25)$. Previous studies have demonstrated that JAK is upregulated in NSCLC and advances the proliferation of NSCLC cells (26). Gao et al found that JAK accelerates the progression of NSCLC and leads to poor prognosis (27). However, the relationship has not been explored between COL6A6 and the JAK signalling pathway. GSEA results showed that the JAK signalling pathway was remarkably concentrated in the COL6A6 low expression group.

Further research revealed that knockdown of COL6A6 increased the p-JAK and p-STAT levels in NSCLC cells. We therefore demonstrated that COL6A6 might inhibit the proliferation and invasion of NSCLC cells by regulating the JAK signalling pathway. Our study demonstrated the relationship between COL6A6 and the JAK signalling pathway, but it has not been confirmed specifically through in vivo experiments, and further experimental studies are needed to support these findings.

In conclusion, our study demonstrated that COL6A6 is a novel tumor suppressor gene in NSCLC and can serve as a potentially valuable prognostic biomarker and therapeutic target for NSCLC.

\section{Acknowledgments}

Funding: This work was supported by the development of Class A critical disciplines by the Qingdao Healthy Committee.

\section{Footnote}

Reporting Checklist: The authors have completed the MDAR reporting checklist. Available at https://dx.doi. org/10.21037/tcr-21-2002

Data Sharing Statement: Available at https://dx.doi. org/10.21037/tcr-21-2002

Conflicts of Interest: All authors have completed the ICMJE uniform disclosure form (available at https://dx.doi. org/10.21037/tcr-21-2002). The authors have no conflicts of interest to declare.

Ethical Statement: The authors are accountable for all aspects of the work in ensuring that questions related to the accuracy or integrity of any part of the work are appropriately investigated and resolved. The Ethics Committee of Qingdao Municipal Hospital Affiliated to Qingdao University approved this study (2021yxy32). All procedures involving human participants in this study are consistent with the Helsinki Declaration (as revised in 2013). Informed consent was obtained from patients.

Open Access Statement: This is an Open Access article distributed in accordance with the Creative Commons Attribution-NonCommercial-NoDerivs 4.0 International License (CC BY-NC-ND 4.0), which permits the noncommercial replication and distribution of the article with the strict proviso that no changes or edits are made and the original work is properly cited (including links to both the formal publication through the relevant DOI and the license). See: https://creativecommons.org/licenses/by-nc-nd/4.0/.

\section{References}

1. Siegel RL, Miller KD, Jemal A. Cancer statistics, 2020. CA Cancer J Clin 2020;70:7-30.

2. Ferlay J, Colombet M, Soerjomataram I, et al. Cancer statistics for the year 2020: An overview. Int J Cancer 2021. [Epub ahead of print]. doi: 10.1002/ijc.33588.

3. Oser MG, Niederst MJ, Sequist LV, et al. Transformation from non-small-cell lung cancer to small-cell lung cancer: molecular drivers and cells of origin. Lancet Oncol 2015;16:e165-72.

4. Hirsch FR, Suda K, Wiens J, et al. New and emerging targeted treatments in advanced non-small-cell lung cancer. Lancet 2016;388:1012-24.

5. Hirsch FR, Scagliotti GV, Mulshine JL, et al. Lung cancer: current therapies and new targeted treatments. Lancet 2017;389:299-311.

6. Siegel RL, Miller KD, Goding Sauer A, et al. Colorectal cancer statistics, 2020. CA Cancer J Clin 2020;70:145-64.

7. Herbst RS, Soria JC, Kowanetz M, et al. Predictive correlates of response to the anti-PD-L1 antibody MPDL3280A in cancer patients. Nature 2014;515:563-7.

8. Hahn WC, Bader JS, Braun TP, et al. An expanded universe of cancer targets. Cell 2021;184:1142-55.

9. Voiles L, Lewis DE, Han L, et al. Overexpression of type VI collagen in neoplastic lung tissues. Oncol Rep 
2014;32:1897-904.

10. Fitzgerald J, Rich C, Zhou FH, et al. Three novel collagen VI chains, alpha4(VI), alpha5(VI), and alpha6(VI). J Biol Chem 2008;283:20170-80.

11. Long R, Liu Z, Li J, et al. COL6A6 interacted with P4HA3 to suppress the growth and metastasis of pituitary adenoma via blocking PI3K-Akt pathway. Aging (Albany NY) 2019;11:8845-59.

12. Yeh MH, Tzeng YJ, Fu TY, et al. Extracellular Matrixreceptor Interaction Signaling Genes Associated with Inferior Breast Cancer Survival. Anticancer Res 2018;38:4593-605.

13. Willumsen N, Bager C, Karsdal MA. Matrix Metalloprotease Generated Fragments of Type VI Collagen Have Serum Biomarker Potential in Cancer - A Proof of Concept Study. Transl Oncol 2019;12:693-8.

14. Holm Nielsen S, Mortensen JH, Willumsen N, et al. A Fragment of Collagen Type VI alpha-3 chain is Elevated in Serum from Patients with Gastrointestinal Disorders. Sci Rep 2020;10:5910.

15. Kang CY, Wang J, Axell-House D, et al. Clinical significance of serum COL6A3 in pancreatic ductal adenocarcinoma. J Gastrointest Surg 2014;18:7-15.

16. Feng Y, Guo X, Tang H. SLC6A8 is involved in the progression of non-small cell lung cancer through the Notch signaling pathway. Ann Transl Med 2021;9:264.

17. Groulx JF, Gagné D, Benoit YD, et al. Collagen VI is a basement membrane component that regulates epithelial cell-fibronectin interactions. Matrix Biol 2011;30:195-206.

18. Naba A, Pearce OMT, Del Rosario A, et al. Characterization of the Extracellular Matrix of Normal and Diseased Tissues Using Proteomics. J Proteome Res 2017;16:3083-91.

19. Castagnaro S, Gambarotto L, Cescon M, et al. Autophagy in the mesh of collagen VI. Matrix Biol 2021;100-101:162-72.
20. Sirisena ND, Samaranayake UMJE, Neto OLA, et al. A novel variant in the COL6A1 gene causing Ullrich congenital muscular dystrophy in a consanguineous family: a case report. BMC Neurol 2021;21:105.

21. Nissen NI, Kehlet S, Boisen MK, et al. Prognostic value of blood-based fibrosis biomarkers in patients with metastatic colorectal cancer receiving chemotherapy and bevacizumab. Sci Rep 2021;11:865.

22. Wang PP, Ding SY, Sun YY, et al. MYCT1 Inhibits the Adhesion and Migration of Laryngeal Cancer Cells Potentially Through Repressing Collagen VI. Front Oncol 2020;10:564733.

23. Hong YG, Xin C, Zheng H, et al. miR-365a-3p regulates ADAM10-JAK-STAT signaling to suppress the growth and metastasis of colorectal cancer cells. J Cancer 2020;11:3634-44.

24. Yang Y, Zhang Q, Liang J, et al. STAM2 knockdown inhibits proliferation, migration, and invasion by affecting the JAK2/STAT3 signaling pathway in gastric cancer. Acta Biochim Biophys Sin (Shanghai) 2021;53:697-706.

25. Li C, Cao Y, Zhang L, et al. LncRNA IGFBP4-1 promotes tumor development by activating Janus kinase-signal transducer and activator of transcription pathway in bladder urothelial carcinoma. Int J Biol Sci 2020;16:2271-82.

26. Baumgartner U, Berger F, Hashemi Gheinani A, et al. miR-19b enhances proliferation and apoptosis resistance via the EGFR signaling pathway by targeting PP2A and BIM in non-small cell lung cancer. Mol Cancer 2018;17:44.

27. Gao Y, Luo L, Xie Y, et al. PYCR1 knockdown inhibits the proliferation, migration, and invasion by affecting JAK/ STAT signaling pathway in lung adenocarcinoma. Mol Carcinog 2020;59:503-11.

(English Language Editor: C. Betlzar)
Cite this article as: Qiao H, Feng Y, Tang H. COL6A6 inhibits the proliferation and metastasis of non-small cell lung cancer through the JAK signalling pathway. Transl Cancer Res 2021;10(10):4514-4522. doi: 10.21037/tcr-21-2002 